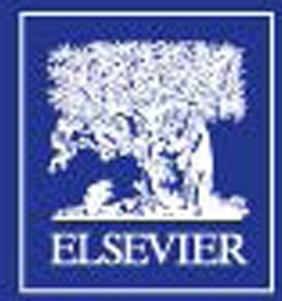

ISSN0924.9338

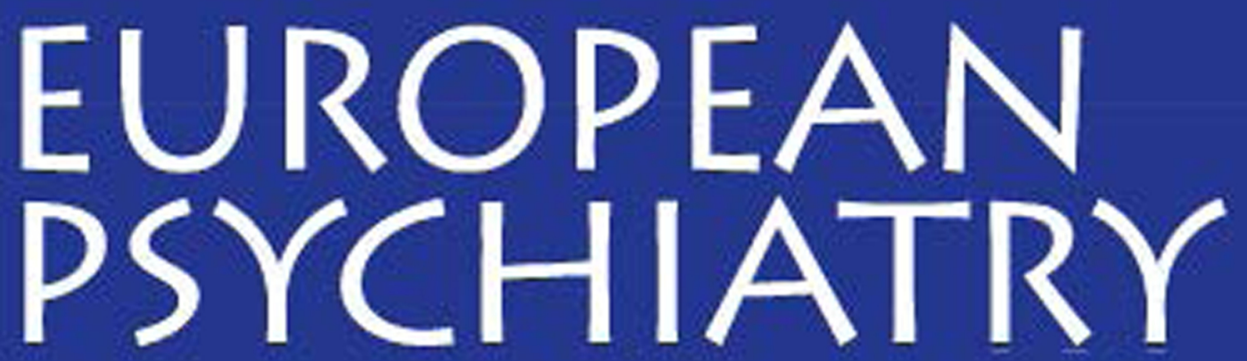

THE IOURNAL OF THE ASSOCIATION OF EUROPEAN PSYCHIATRISTS

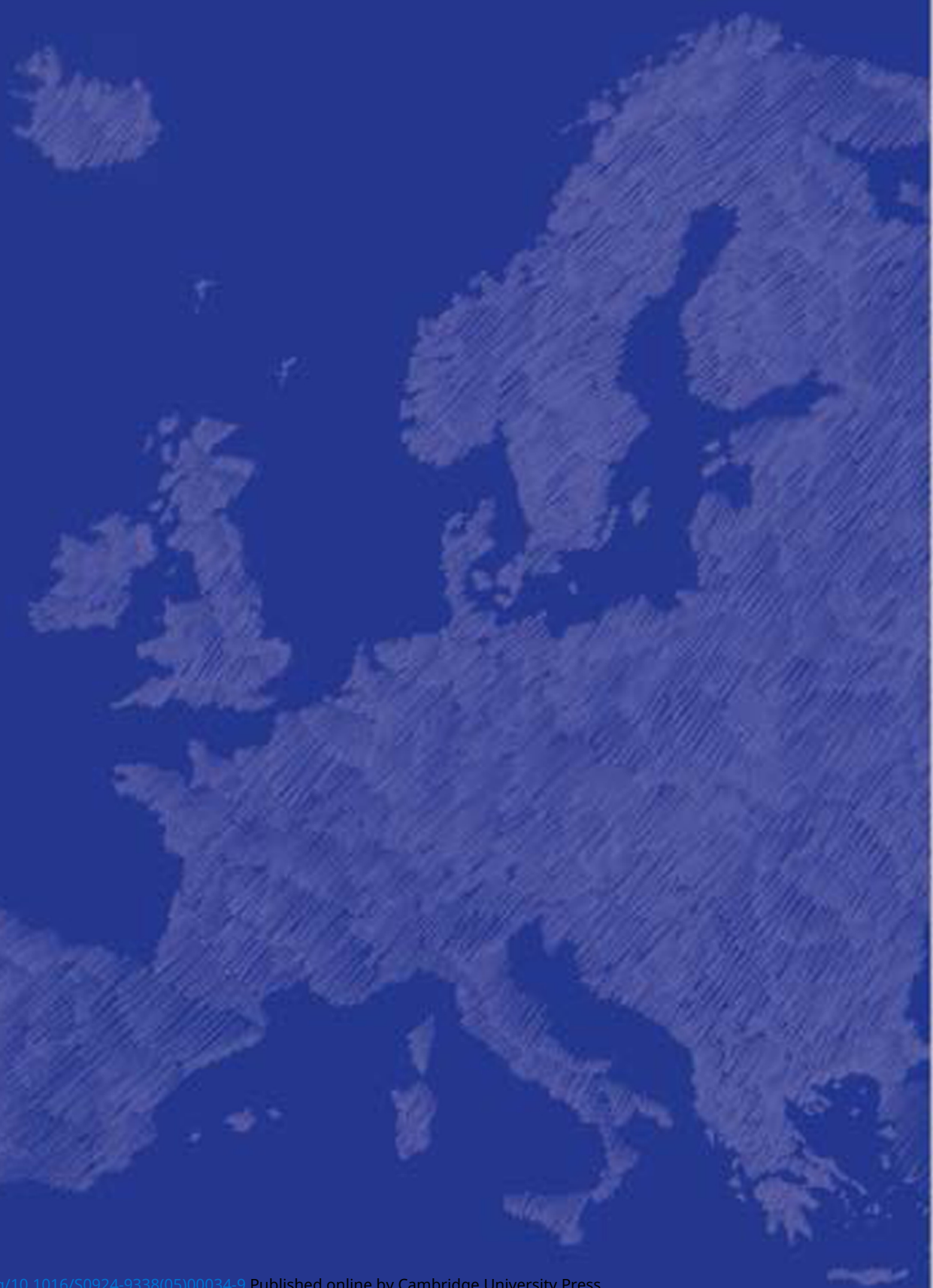

85 Letter from the AEP President BIPOLAR

87 Update on the trestment of bipolar disorder in children and adolescents R.L. Finthe

92. Nea data on the uso of Bthium, fivalproate. and lamotrigine in rapid cycing bipolar disorder

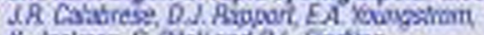

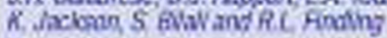

96 The treatment of mixed states and the risk of ssitcling to depression E. Wits

SUICIDE

101 Prediction of repeated parasuicide after $1-12$ months

ik. Cederole and A Gerwasn

110 Sulcide in Cyprus 1968-1999

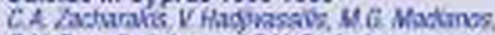

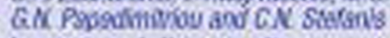

115 Long-term follow-up alter severe suicide attemet by multiplo blunt trauma E-Ge Ponc S Rectrotz. C. Whych a and r. Sctuador Axman

121 Effect of a Suicide Prevention Centre for young people with suicidal behaviour in Copenhagen

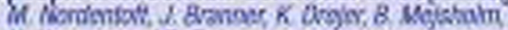
H. Hassen and B Provssan

129 Nicotine use in suicides: a case-controi study B. Sennider A Schnad B. Weoer L fickeh, $K$. Maurer and r. Wetherling TRAUMA

137 Earthquake-telaled psychological diatress and associated faclors 4 years after the Parnitha earthquake in Greoce

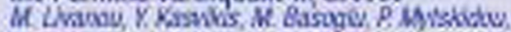

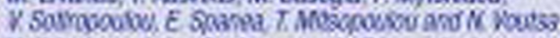

145 Validation of the Peritraumstic Dissociatve Experiences Ouestiennsire sell-report version in Iwo samples of French-spsaking individuals exoosed to trauna

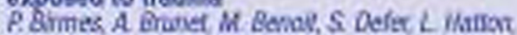
$H$ Secolnsin and $L$ Schumer

152 Etlects of internal displacement and resettlement on the trentsi health of Turkish children and adolesocnts N. ErW, Z. Simsok D. Oner aso K Mat

158. Posttraumatic stross disorder and psychiatric co-morbidity: symptoms in a random semple of feesale Bosnian rafugees $K$ S.ndzust L.W. Sohonsson, V. Dovtratva S.E Jhanssco ad I Sindaust

\title{
EATING DISORDEAS
}

I65 Predictability of a favorable outcoose in anorexia nervos: HC Detor O Scristorg W Noga. HC. Fitederich and W Rorwy

173 Near contributions to the prevalenos of eating cisorders in Spanish adolescents: detection of false negativgs

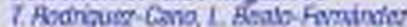
aNA Botavor lanto

179 Incidense of eating esonders in Naratra (Spain)

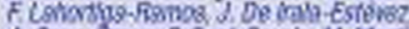

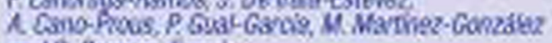
aw'S. Comwo Gngul

186 Modified nutritional counsel ing to increase motivation to treatment in anorevia nervosa

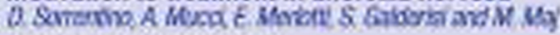
MISCELLANEOUS

188 Temporament and character in primary insomnia $\angle$ de Satur baste, 1 Stravo and $A$ Folssabo

is3. Moroccan colloquial Arabic version of the Mini International Nouropsychiatric Intervieu (MIND): qualitative and quantifative validation

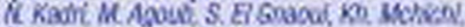
7. kerquers and D. Moussake

196 CORRIGENDUM 


\title{
EUROPEAN PSYCHIATRY \\ THE JOURNAL OF THE ASSOCIATION OF EUROPEAN PSYCHIATRISTS
}

\section{EDITORS}

S. Frangou (London) \& Ph. Gorwood (Paris) \& R. Heun (Bonn/Durham).

Editorial Office : Prof. Ph. Gorwood, Editor of European Psychiatry - INSERM U675, Faculté Xavier Bichat, 16, rue Henri Huchard,

75018 Paris (France). e-mail : europsy@free.fr - Tel. 33130430250 - Fax. 33130431762.

\section{EDITORIAL BOARD}

P. Boyer (Ottawa), J.D. Guelfi (Paris), M. Maj (Naples), R. Murray (London), H. Sass (Aachen)

\section{STATISTICAL ADVISORS}

A. Heyting (Da Marken), N. Takei (Hamamatsu), B. Falissard (Paris)

\begin{abstract}
ADVISORY BOARD (permanent reviewers)
Aalto-Setälä, Terhi, Espoo, Finland; Amaddeo, Francesco, Verona, Italy; Arango, Celso, Madrid, Spain; Barbui, Corrado, Verona, Italy; Bellivier, Franck, Créteil, France; Bertschy, Gilles, Geneva, Switzerland; Brambilla, Paolo, Milan, Italy; Broome, Matthew, London, UK; Clark, Luke, Cambridge, UK; Clarke, Mary, Stillorgan, Ireland; Colom, Francesc, Barcelona, Spain; Courtet, Philippe, Montpellier, France; Craddock, Nick, Birmingham, UK; Crocq, Marc-Antoine, Rouffach, France; Dazzan, Paola, London, UK; Delamillieure, Pascal, Caen, France; Deuschle, Michael, Mannheim, Germany; Dollfus, Sonia, Caen, France; Dubertret, Caroline, Colombes, France; Ekselius, Lisa, Uppsala, Sweden; Fabrozzo, Michele, Naples, Italy; Falkai, Peter, Hamburg, Germany; Favaro, Angela, Padova, Italy; Galderisi, Silvana, Naples, Italy; Garcia-Portilla, Maria-Paz, Oviedo, Spain; Giannakopoulos, Panteleimon, Geneva, Switzerland; Gonzales-Pinto, Ana, Vitoria, Spain; Gourion, David, Paris, France; Grabe Hans-Jörgen, Stralsund, Germany; Gustavsson, Petter, Stockholm, Sweden; Hansenne, Michel, Liege, Belgique; Heinz, Andreas, Berlin, Germany; Hosak, Ladislav, Hradec Kralove, Czech Republic; Hrdlicka, Michal, Prague, Czech Republic; Kircher, Tilo, Auchen, Germany; Kirov, George, Cardiff, UK; Kontaxakis, Vassili, Athens, Greece; Kurzthaler, Ilsemarie, Innsbruck, Austria; Lederbogen, Florian, Mannheim, Germany; Licht, Rasmus-Wentzer, Risskov, Denmark; Lieb Roselind, Munich, Germany; MacCabe, James, London, UK; MacGregor-Lawrie, Stephen, Edimburgh, Scotland; Mallet, Luc, Paris, France; Martinot, Jean-Luc, Orsay, France; McDonald, Colm, London, UK; McGuire, Philip, London, UK; Menchon, Jose-Manuel, Hospitalet de Llobregat, Spain; Mico, Juan-Antonio, Cadiz, Spain; Mohr, Pavel, Prague, Czech Republic; Monteleone, Palmiero, Naples, Italy; Mucci, Armida, Naples, Italy; Müller, Ulrich, Cambridge, UK; Murray, Graham, Cambridge, UK; Neeleman, Jan, Groningen, the Netherlands; Nordström, Anna-Lena, Stockholm, Sweden; O'Callaghan, Eadbhard, Dublin, Ireland; Öngur, Dost, Boston, USA; Oswald, Pierre, Brussels, Belgium; Paillere-Martinot, Marie-Laure, Paris, France; Papadimitriou, George, Athens, Greece; Pariante, Carmine M., London, UK; Pélissolo, Antoine, Paris, France; Percudani, Mauro, Milan, Italy; Perez, Jorge, Brescia, Italy; Pilowski, Lyn, London, UK; Pini, Stefano, Pisa, Italy; Pinto, Emmanuel, Liege, Belgium; Pitchot, William, Bertrix, Belgium; Runeson, Bo, Stockholm, Sweden; Sahakian, Barbara, Cambridge, UK; Schmidt, Ulrike, London, UK; Schuman, Gunther, Mannheim, Germany; Schütz, Christian, Bonn, Germany; Schwarz, Marcus, Munich, Germany; Sommer, Wolfgang, Stockholm, Sweden; Souery, Daniel, Brussels, Belgium; Spence, Sean A., Sheffield, UK; Ströhle, Andreas, Berlin, Germany; Suvisaari, Jaana, Helsinki, Finland; Thome, Johannes, Swansea, UK; Tortorella, Alfonso, Naples, Italy; Van Os, Jim, Maastricht,

The Netherlands; Verdoux, Hélène, Bordeaux, France; Weiss, Elisabeth M., New York, USA; Yazgan, Yanki, Istanbul, Turkey.
\end{abstract}

\section{EDITORS EMERITUS}

C. Ballus (Barcelona), P. Bech (Copenhagen), H. Heimann (Tübingen), Y. Lecrubier (Paris), C.B. Pull (Luxembourg)

THE JOURNAL

OF THE

ASSOCIATION OF EUROPEAN PSYCHIATRISTS

www.aep.lu
President: H. Sass (Aachen)

Past President: M. Maj (Naples)

President Elect: C. Höschl (Prague)

Secretary General: M. Kastrup (Copenhagen)

Treasurer: K. Mann (Mannheim)

Counsellors: P. Boyer (Ottawa), S. Tyano (Petach-Tikva)

Sections: M. Musalek (Vienna) 